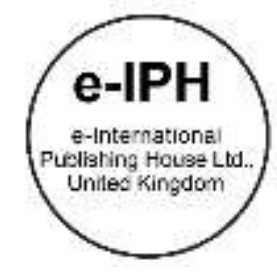

\title{
Public Perceptions and Behaviours towards Bicycle Sharing Offered by SMEs as a Mode of Transportation and the Way Forward
}

\author{
Herwina Rosnan ${ }^{1}$, Nuraisyah Chua Abdullah² \\ ${ }^{1}$ Arshad Ayub Graduate Business School \\ ${ }^{2}$ Faculty of Law, \\ Universiti Teknologi MARA, 40450 Shah Alam Selangor, Malaysia \\ herwinaros@gmail.com, nuraisyahc@yahoo.com, nuraisyah@salam.uitm.edu.my \\ Tel: +60192345680
}

\begin{abstract}
Bicycle sharing has gained popularity across the world especially in developed countries. Bicycle sharing is a non-polluting and healthy mode of transportation. Compared to motorised mode of transportation, bicycle sharing is seen as a good alternative for the environment. Particularly in urban areas where pollution from vehicles' emission is a concern. Bicycle sharing is relatively new to Malaysian citizens. Hence, the primary objective of the study is to examine public perceptions towards bicycle sharing. Primary and secondary data was collected and analysed. The findings indicate that the public do not really appreciate the concept of bicycle sharing. Policy can be directed towards supporting bicycle sharing.
\end{abstract}

Keywords: Bicycle sharing; Public transportation; Quality of life; Malaysia

eISSN: 2398-4287 @ 2018. The Authors. Published for AMER ABRA cE-Bs by e-International Publishing House, Ltd., UK. This is an open access article under the CC BYNC-ND license (http://creativecommons.org/licenses/by-nc-nd/4.0/). Peer-review under responsibility of AMER (Association of Malaysian Environment-Behaviour Researchers), ABRA (Association of Behavioural Researchers on Asians) and cE-Bs (Centre for Environment-Behaviour Studies), Faculty of Architecture, Planning \& Surveying, Universiti Teknologi MARA, Malaysia.

DOI: https://doi.org/10.21834/e-bpj.v3i8.1411

\subsection{Introduction}

A bicycle sharing system is a network of bicycles, which is also known as cycle-hire, public bicycle system, bike-share and cycle-share. Globally, bicycle sharing has become popular as an alternative to motorised public transport. Currently, the concept of bicycle sharing system is available in more than 200 countries. In many instances, bicycle sharing is promoted for short distance destination that connects the users to other public transport. In other words, bicycle sharing is associated with other public transport network it serves as a connection for users to other public transportation such as train service. According to Wine (2012), a bicycle sharing system is a "network of bicycles" and kiosks that the public which include tourists and students can grab for a short distance trip and then return it to any designated station or dock in different location. Harrah (2012) further explain that bicycle sharing system refers to bicycles which are not owned by individuals but service providers or operators that is placed in major cities, town, campuses or commercial areas that enable the community to access the service based on stipulated cost, time, distance of the trip as set by the operators or bicycle sharing service provider.

Although bicycle transport has long been practised in many countries, the concept of bicycle sharing is relatively new. According to DeMaio and Meddin (2012), in many urban areas, as a commercial transport system, bicycle sharing system is indeed a relatively new practice. Renting a bicycle through bicycle sharing concept is a challenge to bicycle sharing service operators as many instances indicate that without proper management bicycle sharing is highly subject to public vandalism. Thanks to technology, many bicycle sharing operators have come up with various technologies to protect the businesses and increase safety of the passengers. This include using

eISSN: 2398-4287 @ 2018. The Authors. Published for AMER ABRA cE-Bs by e-International Publishing House, Ltd., UK. This is an open access article under the CC BYNC-ND license (http://creativecommons.org/licenses/by-nc-nd/4.0/). Peer-review under responsibility of AMER (Association of Malaysian Environment-Behaviour Researchers), ABRA (Association of Behavioural Researchers on Asians) and cE-Bs (Centre for Environment-Behaviour Studies), Faculty of Architecture, Planning \& Surveying, Universiti Teknologi MARA, Malaysia.

DOI: https://doi.org/10.21834/e-bpj.v3i8.1411 
Global Position System (GPS) and Radio Frequency Identification (RFID) to track the down the bicycles. Automation is now a backbone of the business with many features to increase the ease of use. In 1998, France was the first country which introduced the first automated system by incorporating information and technology (ICT). The use of smart card makes it convenient for user to collect the bicycle and easy drop-off at the station. Now, bicycle sharing is also referred to as 'Smart Bikes", "Bicycle Transit" and Public-Use Bicycle.

The concept of bicycle sharing system involves rental scheme of bicycles where user can collect the bicycle at any self-service station and leave it at any other bicycle dock designated by the service provided. This concept is similar to many car rental service except that bicycle sharing is meant for a short distance trip. The attraction of bicycle sharing concept is that users do not have to won a bicycle to commute and bicycle sharing provides a relatively cheaper and affordable mode of public transport.

Since it was first introduced, bicycle sharing has moved from merely a new exciting concept to some mainstream public transport alternatives. It has been widely used in major cities like London and Paris. It is estimated that there are 375 different bicycle sharing scheme all over the world and regionally there are around 236,000 usage of bicycle sharing with the largest consumption is in Hangzhou, China where 40,000 bicycle sharing usage was recorded (Wine, 2012).

\subsection{The Evolution of Bicycle Sharing}

Since the introduction of bicycle sharing in the 1960s in Europe, there was a dramatic change in its service offerings. Bicycle sharing started in Amsterdam in 1965 followed by La Rochelle in 1976 and later bicycle sharing is introduced in Cambridge in 1993. These are the location where the concept of bicycle sharing in which user can borrow and return the bicycle at any location was introduced. However, in the early operations, there were no measures were taken to ensure the bicycles are used and cared of. Because of many cases of vandalism and theft, makes the service offerings unattractive to the operators. In 1991, a second generation of bicycle sharing was introduced in Denmark that able to overcome the issue of theft and vandalism (DeMaio, 2009). Then the third generation was later introduced in 1995, where the bicycle sharing services was offered in Copenhagen on a large scale. By 1995, the first large scheme was introduced in Copenhagen. The new scheme emulated "lending library" scheme with an annual fee or membership fee charge to the users (Metrolinx, 2009) with a membership or annual fee. In order to reduce theft, the service provider or operators built a nonstandard component. The bicycle was also a heavy duty bicycle compared to normal bicycle to withstand frequent usage. The new technology built was simple and cheaper to install. Some operators used manual tracking but most used coin-operated locks to locked the bicycle. Although the system was better than the previous system, it was only able to reduce theft and vandalism. Hence, in 1990s, a more reliable system of smartcard was introduced with a more reliable features to protect the interest of the operators and the safety of the passengers. France was the first location that introduced the use of smartcard technology in its bicycle sharing schemes in 1998 followed by France in 2007. These third generation of bicycle sharing are more sophisticated in that it is not only improve in the bicycle features but also the use of automatic features for payment systems and bicycle locking. Global Positioning System (GPS) and Radio Frequency Identification (RFID) technologies were among popular system that enable operators to track down the bicycles which ultimately control vandalism and theft. Websites are also important tools to provide real time information on the availability of the bicycle at any stations. In most instances, bicycle sharing service is a collaboration effort of private and public partnership.

\subsection{Bicycle Sharing in Malaysia}

Bicycle sharing system was introduced in Penang as an alternative mode of getting around Georgetown (a heritage city) without driving. The intention of the service is to help in reducing the number of cars on the road within the popular tourist attraction. Further, the development of Malaysian economy is an opportunity for the establishment of bicycle sharing service business. With the advancement of public transportation systems in Malaysia, makes the concept of bicycle sharing relevant to be introduced to help the users to access to the public transit network. Liu, Jia and Cheng (2012) terms the new solution as "the last mile solution". The concept of bicycle sharing can now be found in Kuala Lumpur, Selangor, Johor and Terengganu. In 2017, more foreign bicycle sharing system operators entered the Malaysian market.

However, bicycle sharing is a new concept in Malaysia, and unlike other countries, the readiness and acceptance of bicycle sharing concept has yet to be known. Companies like O.Bike found it challenging to serve the new market as the understanding, culture and awareness of the public are not known. In fact, cycle culture in Malaysia is usually for leisure activity purposes and sports and not as a mode of transportation. Thus, the primary objective of this study is to seek understanding on public perceptions towards bicycle sharing as a mode of transportation. This study is an exploratory study where data is gathered mainly to provide in-depth understanding of the current practice of bicycle sharing in Malaysia.

\subsection{Literature Review}

Bicycle sharing has received scholar attentions since the new schemes was first introduced. However, there were conflicting findings on the success of bicycle sharing on users, business operators and the cities in general. For example, Study by Liu et. all (2012) in Beijing city found a declining popularity of bicycle sharing, while study by Roman and Roman (2014) in Warsaw city found an increasing prevalence and increase number of usage. However, the fact the bicycle sharing system posed positive characteristic particularly in promoting sustainable mobility, the exploitation of bicycle sharing business is still attractive (Bordagaray, Ibeas and Olio, 2012).

In a country where bicycle sharing concept is new, studies were focus on the perception of the public or users on bicycle sharing services (for example, Bordayaray et al., 2012 and Sousa et al., 2014). The concept of bicycle sharing is deemed beneficial to all in that is is good for the environment (non-pollutant), good for the users (healthy lifestyle) and good for the cities (less congestion on motorised traffic). However, there are indeed many barriers to the usage of bicycle. Sousa et al. (2014) found that the infrastructure plays an 
essential factor for bicycle sharing usage. The inadequacy of dedicated bicycle infrastructure posed a significant barrier that hinder usage of bicycle sharing in Brazil. The study also found that the perceived barrier differs between cities in which slopes become the second critical barrier in Sao Paulo city while security and climate was the second crucial barrier for Campo Grande city. Although infrastructure was found as an essential factor that discourage bicycle sharing, it was beyond the capability of business operators to handle it as the local government plays an important role to ensure proper infrastructures and public facilities. This factor is the reason why most countries enter into private-public partnership when it comes to bicycle sharing service offerings. For example, in Germany, Netbike and Norisbike are working together providing self-service bike central to support urban transport as well as to promote urban tourism where German Ministry of Transport supported the project.

Previous studies found barriers that hinder the usage of bicycle sharing includes lack of infrastructure like dedicated bicycle track, safety, weather, geographical landscape, distance of the trip, physical fitness and inconvenience (Fishman, 2012; Sousa et al., 2014). Studies also conducted to identify gender difference in cycling where males were found to be a more frequent to cycle that female. The reason is related to factors like convenience where women are associated with activities like shopping, transporting kid to school where the usage of private car is a necessity. Furthermore, women are also associated with higher conscious towards safety and are more particular about the appearance to work (Garrard et al., 2008). Nevertheless, in country with high level of cycling, there is a balanced number of men and women bicycle cyclists like in the Netherlands (Garrard, 2003). Concerning age, Parkin (2004) found that higher proportion of people who are under 34 years old cycle more. This could be related to physical nature of the younger age, lower level of car ownership factor and the tendency of younger people to live in the cities area.

In a new location where bicycle sharing is a new initiative, scholars conducted studies to investigate the perceptions of the public towards bicycle sharing. In this context, Davis's model (1989) of Technology Adoption) carried two theoretical constructs that is relevant in examining perception of users on bicycle usage. These two constructs are perceived usefulness and perceived ease of use. Both constructs are seen to directly impact the intention to use a technology. Perceived usefulness is related to the people's perception that on how technology facilitates them in carrying out their task. The authors indicate that the intention of users to use system can be measured using these two constructs of perceived ease of use and perceived usefulness as it is suggested that both are predominantly necessary measures (Davis, 1989; Lee, 2010; Vankatesh and Davis, 1996). Hence, TAM is a model that emphasises on perceived ease of use and perceived usefulness as two fundamental determinants of technology acceptance by users (Davis, 1989). The definition of Perceived Ease of Use as defined by Davis (1989) is "the degree to which a person believes that using a particular system would be free of effort" and hypothesized that perceived ease of use has a positive effect on perceived usefulness as "the degree to which a person believes that using a particular system would enhance his or her job performance".

\subsection{Perceived Usefulness}

It has been found in a study of bicycle sharing system program can lead to reduced demand for parking space, reduced effect to greenhouse emission and reduced traffic congestion, thereby helping minimise resource consumption and nurture a greener environment in campus (Ashley, 2012). Furthermore, cycling is associated with a healthy lifestyle and increases the health of a person. It is also linked to the barriers of bicycle sharing usage with the common concerns about the climate, travelling distance, traffic congestion, crowding of cycling path, the use of GPS while cycling and the automatic payment system. According to TAM and other related literature, attitude and a behavioural intention can be fostered by perceived usefulness (Lee, 2015). When people feel that the commercial bicycle sharing systems are useful and beneficial to them, they will develop a positive view toward the bike-sharing system, and be more willing to use the system. Since bicycle sharing is using special application to operate their bicycle, it can relate to the literature that a mobile application (mobile apps) is generally perceived as more useful provided the usage is easy (Wenga et al., 2017). In the other study by Mkpojiogu and Hussain (2016), they describe perceived of usefulness in a context that, the user may accept that a given innovation such as a mobile map is helpful, but while using the mobile, the user may find out that the innovation may be difficult to use. For instance, the object on mobile screen may be difficult to see.

\subsection{Perceived Ease of Use}

Users' intention of technology adoption can be measured by perceived ease of use of the technology (Davis, 1989). This denotes that individuals believe the use of services or products is effortless, that using particular product/service would be free from effort. For example, utilising a specific technology like mobile map would be free of physical and mental exertion. In the same token, Applying the same concept in the context of application technology, perceived ease of use is inferred as the degree to which users perceive the ease of interface with application allows them to obtain appropriate and useful information they required (Rahman et al., 2013). This statement is consistent with Yuslihari et al. (2011) findings which suggested that PEOU has positive and significant influence towards consumers' attitude on online purchasing. Anwarul and Salma (2016) stated that there is a positive causality between PEOU and usage intention. Ease of Use is the user's impression of the measure of requirement needed to use a technology or the level to which user accepts that applying a specific innovation will be easy and effortless (Chen, 2016). Apart from Davis et al., Chen (2016) also included Perceive Ease of Use as precursor to intention to use.

\subsection{Methodology}

This is a qualitative study which main aim is to gain an in-depth understanding of the public perceptions on bicycle sharing. This study is an exploratory study where data collection was conducted through interview. Bicycle sharing businesses in Malaysia are mostly located at universities and train station, hence, respondents from these locations were approached. A total of 15 respondents were 
willing to participate in the study. The interview sessions were recorded and data was transcribed. Most of the interview session lasted less than half an hour as most of the respondents were public transport commuters. The study employed a semi-structured interview. The analysis was done manually by comparing the transcripts to look at similarities and differences of information. Analysis was not done verbatim as the aim of the study is to look for major ideas related to the phenomenon under study. Content analysis was also conducted to analyses secondary data.

\subsection{Discussion}

From the preliminary interview, the majority public in Malaysia do not really appreciate the concept of bicycle sharing as they do not think this is a feasible idea. Moreover, lack of bicycle path makes it inconvenient for public to use the bicycle sharing as one of their main transportation mode which this finding is consistent with previous studies (Fishman, 2012; Sousa et al., 2014). It is difficult for the cyclist to find the correct road to cycle on because bicycle stand at a weird middle ground between transportation and pedestrians. On top of that, the weather is one of the main factors the public is hesitant to use bicycle as mode of transportation. The understanding of the behaviour and perceptions of public towards bicycle sharing would be able to upgrade the SMEs bicycle sharing industry and contributes towards the creation of sustainable city. Policy can be directed into educating and promoting public transportation, which is environmentally friendly.

Bicycle sharing system is uncommon to most Malaysian as it is not a culture to use bicycle as a medium of commuting from places to places. Cycling culture in Malaysia is usually for leisure activities purposes and sports. Besides, hot weather in Malaysia makes public think twice to use bicycle as their main transportation. The general public do not really understand the intention of introducing this bicycle sharing in Malaysia as they do not think that this is an excellent idea for them to use it.

Star Online June 2017 reported that there is an increasing number of bicycle sharing users as indicated in the growing number the bicycle sharing applications. Currently, it is estimated that there are now 20,000 users. The rental is based on usage in which every 15 minits usage the user will be charge RM1. However, the number of users decreasing by 10 percent every quarter of the month since public usually want to give it a try as a first timer and not repeating for the next time. They would instead take Grab, Uber or taxi to their destination rather than using bicycle sharing. The issue of a decreasing number of users is not unique to Malaysia market which is relatively new in bicycle sharing. In a more matured market like Beijing, they are also experiencing a decreasing number of bicycle sharing user as these users prefer other modes of public transport.

Moreover, due to lack of education on the bike sharing system in Malaysia, users do not aware of the parking system of bicycle sharing cause them to park everywhere and disrupt the traffic. According to local mainstream newspaper, The New Straits Times (NSTP) in September 2017 reported that Petaling Jaya City Council (MPAJ) enforcement officers have seized more than 250 bicycles found on the sidewalks and streets of Petaling Jaya (MBPJ). The bicycles were seized because there were obstructions on the road. Further to seizure, the bicycle sharing service provider had been fined RM17,000 by Petaling Jaya City Council (MBPJ). The bicycle sharing has been instructed by the MBPJ to remove all its existing bicycle in Selangor except in Subang Jaya as Subang Jaya will introduce a pilot project for bicycle sharing concept beginning October 2017. MPPJ also claimed that the dockless idea makes the bicycle too messy.

Theft and vandalism are everywhere in the world and Malaysia is not an exception. Malaysian Digest, November 2017 stated that some of the bicycle sharing, bicycles were found in inappropriate places such as thrown in drains and bushes, left in hidden places in housing areas and even hanging from signboards. Vandalism of the bicycles can be found in missing paddles, handles and even tires. This affects the offerings of bicycle sharing service where the service providers have to come up with a more stringent terms of bicycle sharing.

On the impact of the bicycle sharing scheme to the cities, Buhrmann (2007) stated that there is little impact which has been reported. The only noticeable effect would be the increasing number of bicycle sharing schemes. The author also said that in Barcelona, Leon and Paris, bicycle sharing posed little impact on the effort to reduce car on the road. It was noted that the percentage of motorised trip that is replaced by bicycle sharing is merely an average of 2-4 per cent. However, Shereen et al. (2010) reported that the user of bicycle increased by 44 per cent in Lyon when it was first introduced. Meanwhile, Paris is experiencing a 70 per cent increase of user after bicycle sharing was launched. Although there is a limited data the impact of bicycle sharing A survey on user in 2008 revealed that 90 percent of the respondents said that "bicycle sharing allowed them to make trips that would he otherwise been impossible" while 20 percent of respondents agree that with bicycle sharing it reduced their car usage (Shereen et al., 2010).

Bicycle sharing service providers are still facing vandalism issue. Shereen et al. (2010) further reported that in European Counties Paris is experiencing the most number of vandalism cases despite the use of sophisticated technology to reduce the problem. According to the report "within the first two years of operations, nearly the whole system fleet of 20,600 bicycles had to be replaced at the cost of 400 Euros per bicycle. Some 7,800 bicycle were stolen, and 11600 bicycles were vandalised beyond repair." The findings of this research also indicate vandalism as one of the main problems faced by service operators. Attitude of the users is also a challenge that requires the service operators to introduce a more stringent terms and conditions of usage. However, too many rules imposed on the users hampered the ease of use of the bicycle that eventually could draw the users away.

\subsection{Conclusion and Implication}

Bicycle sharing system is a modern public transport scheme that has potential to be developed. It provides a sustainable public transport alternative and plays an integral part in increasing viable transport options in cities. The understanding of the behaviour and perceptions of the public towards bicycle sharing would be able to upgrade the SMEs bicycle sharing service provider and contributes towards the 
creation of a sustainable city. Policy can be directed towards educating and promoting the growth of sustainable and environmental friendly public transport system. In this context, the role of government is critical in ensuring the success of bicycle sharing system. Based on study by Liu et al. (2012) the authors found out that when the China government intervenes and encourage the users to commute by bicycle, the number of bicycle sharing's users in China increased tremendously. This is when the government restricted the used of motorised vehicles to accommodate for the Olympic Games. However, after the event, there was a sharp drop in the number of bicycle commuters which also affect the business of the service providers. Some of the bicycle sharing service operators had to close down their business as users prefer other modes of public transport.

The benefit of bicycle sharing system should not be overrated. Although theoretically, it is right in terms of reducing congestion, healthy lifestyle of the users and environmental mode of transport, the cost of establishing the system need to be deliberated. The local administrative authority plays essential factor in supporting the initiative. This is because a successful model of bicycle sharing system take a lot more than merely promoting its advantages. For example, in Netherlands, the bicycle sharing system is integrated into a more comprehensive public transport than increase efficiency of bicycle usage. This model integration (Britton, 2009) is a self-promoting tools to increase the utilisation of bicycle sharing.

\section{Acknowledgements}

The authors would gratefully acknowledge the Institute of Research Management and Innovation (IRMI) Universiti Teknologi MARA (UiTM), Shah Alam and Ministry of Higher Education Malaysia (MOHE) for the financial support through the Fundamental Research Grant Scheme, File No: 600-IRMI/FRGS 55/3(36/2015).

\section{References}

Bordagaray, M., Ibeas, A., \& dell'Olio, L. (2012). Modeling user perception of public bicycle services. Procedia-Social and Behavioral Sciences, 54, $1308-1316$.

Britton E. 2009. Public Bikes in Latin American Cities: great idea but what next?

Bührmann, S. (2008). Bicycles as public-individual transport-European developments. Köln, Rupprecht Consult Forschung und Beratung GmbH.

Davis, F. D. (1985). A technology acceptance model for empirically testing new end-user information systems: Theory and results (Doctoral dissertation, Massachusetts Institute of Technology).

Davis, F. D., Bagozzi, R. P., \& Warshaw, P. R. (1989). User acceptance of computer technology: a comparison of two theoretical models. Management Science, 35(8), 982-1003.

de Sousa, A. A., Sanches, S. P., \& Ferreira, M. A. (2014). Perception of barriers for the use of Bicycles. Procedia-Social and Behavioral Sciences, 160, 304-313.

Fishman, E. (2011). Evaluating the benefits of public bicycle schemes needs to be undertaken carefully. British Medical Journal, 343(d4521).

Franco, L. P. C., Campos, V. B. G., \& Monteiro, F. B. (2014). A characterisation of commuter bicycle trips. Procedia-Social and Behavioral Sciences, $111,1165-1174$.

Garrard, J., Rose, G., \& Lo, S. K. (2008). Promoting transportation cycling for women: the role of bicycle infrastructure. Preventive medicine, 46(1), 55-59.

Harrah, K. (2012). Utilizing bicycles to promote physical activity. VAHPERD Journal, 33(2), 4-6.

Horton, D., Rosen, P., \& Cox, P. (Eds.). (2016). Cycling and society. Routledge.

Lammers, J., \& Stapel, D. A. (2011). Power increases dehumanization. Group Processes \& Intergroup Relations, 14(1), 113-126.

Liu, Z., Jia, X., \& Cheng, W. (2012). Solving the last mile problem: Ensure the success of public bicycle system in Beijing. Procedia-Social and Behavioral Sciences, 43, 73-78.

Meddin, R. (2011). The bike-sharing world: First days of summer 2011. 2011 [2012-12-12]. http://bike-sharing. blogspot com/search.

Meddin, R., \& DeMaio, P. (2014). The bike-sharing world: First week of June 2011. 2011 [2012-12-12]. http://bike-sharing. blogspot. com.

METROLINX (2009). Bike Share Program Investigation - Best Practices Investigation. Toronto.

Midgley, P. (2011). Bicycle-sharing schemes: enhancing sustainable mobility in urban areas. United Nations, Department of Economic and Social Affairs, 1-12.

Wardman, M., Tight, M., \& Page, M. (2007). Factors influencing the propensity to cycle to work. Transportation Research Part A: Policy and Practice, 41(4), 339-350.

Parkin, J. (2004) Determination and Measurement of Factors Which Influence Propensity to Cycle to Work. PhD Thesis, Institute for Transport Studies, University of Leeds.

Roman, M., \& Roman, M. (2014). Bicycle transport as an opportunity to develop urban tourism-Warsaw example. Procedia-Social and Behavioral Sciences, 151, 295301. 
Shaheen, S., Guzman, S., \& Zhang, H. (2010). Bikesharing in Europe, the Americas, and Asia: past, present, and future. Transportation Research Record: Journal of the Transportation Research Board, (2143), 159-167.

Vogel, P., Greiser, T., \& Mattfeld, D. C. (2011). Understanding bike-sharing systems using data mining: Exploring activity patterns. Procedia-Social and Behavioral Sciences, 20, 514-523.

Wine, J., \& Easy, B. (2012). Bicycle Share Feasibility Study New Orleans. 